\title{
Quantum random walk of the field in an externally driven cavity
}

\author{
G. S. Agarwal* and P. K. Pathak* \\ Department of Physics, Oklahoma State University, Stillwater, Oklahoma 74078, USA \\ (Received 13 April 2005; revised manuscript received 20 June 2005; published 16 September 2005)
}

\begin{abstract}
Using a resonant interaction between atoms and the field in a high-quality cavity, we show how to realize quantum random walks as proposed by Aharonov et al. [Phys. Rev. A 48, 1687 (1993)]. The atoms are driven strongly by a classical field. Under conditions of strong driving we could realize an effective interaction of the form $i S^{x}\left(a-a^{\dagger}\right)$ in terms of the spin operator associated with the two-level atom and the field operators. This effective interaction generates displacement in the field's wave function depending on the state of the two-level atom. Measurements of the state of the two-level atom would then generate an effective state of the field. Using a homodyne technique, the state of the quantum random walker can be monitored.
\end{abstract}

DOI: 10.1103/PhysRevA.72.033815

PACS number(s): 42.50.Pq, 03.67.Lx

\section{INTRODUCTION}

In a very interesting paper Aharonov et al. [1] proposed the idea of a quantum random walk. Here a random walker is constrained to move left or right depending on the state of an auxiliary quantum-mechanical system. One then examines the state of the random walker subject to the measurement of the state of the auxiliary system. As an interesting consequence of this quantum random walk, Aharonov et al. [1] found that the walker's distribution could shift by an amount which could be larger than the width of the initial distribution. Further the displacement could be much larger than the classical displacement. Several proposals [1-8] exist for realizations of the quantum random walk. For example, Aharonov et al. gave a cavity QED model where the photon number distribution can get displaced. Sanders et al. [2] considered a dispersive interaction in the cavity of the form $S^{z}\left(a+a^{\dagger}\right)$ and considered the random walk of the field on states on a circle. Other interesting theoretical schemes for implementing quantum walks have been suggested in ion traps [4] and in optical lattices [5]. Knight et al. [6] further showed that an earlier experiment [7] was a realization of quantum random walks. A scheme using linear optical elements has been recently implemented [8].

Here we propose a method which yields precisely quantum random walk as proposed by Aharonov et al. We use cavity QED; however, we drive the atoms by an external field. Currently there is considerable progress in realizing a variety of high-quality cavities and a variety of interactions, and thus one is in a situation where proposals like the one presented here are likely to be implemented.

The organization of the paper is as follows. In Sec. II we present the details of our model and show the conditions under which such a model gives rise to an effective Hamiltonian which we use in Sec. III to realize the quantum random walk. In this section we also present the results for the Wigner function for the state of the quantum walker. In Sec. IV we show how the homodyne measurements of the field

*On leave from Physical Research Laboratory, Navrangpura, Ahmedabad-380 009, India. can be used to check the characteristics of the quantum random walk. In Sec. V we incorporate the effects of decoherence due to the decay of the field in the cavity. In the Appendix we discuss the state of the walker if no conditional measurements are made and establish a relation to classical random walks.

\section{EFFECTIVE HAMILTONIAN FOR A QUANTUM RANDOM WALK USING DRIVEN ATOMS}

We consider a two-level Rydberg atom having its higherenergy state $|e\rangle$ and lower-energy state $|g\rangle$ interacting with a single mode of the electromagnetic field in a cavity. The atom passes through the cavity and interacts resonantly with the field. Further the atom is driven by a strong classical field. For simplicity we choose the atomic transition frequency, the cavity frequency, and the frequency of the driving field to be same. The Hamiltonian for the system in the interaction picture is written as

$$
H=-i \hbar g\left(S^{+} a-a^{\dagger} S^{-}\right)+\hbar\left(S^{+} \mathcal{E}+S^{-} \mathcal{E}^{*}\right),
$$

where $g$ and $\mathcal{E}$ are the coupling constants of the interaction of the atom with the cavity field and with the driving field. We have chosen $g$ as real and $\mathcal{E}$ as complex. The annihilation (creation) operator for the field in the cavity is $a\left(a^{\dagger}\right)$, and $S^{+}$ and $S^{-}$are atomic spin operators. The last term in Eq. (1) is the interaction with the external field. We further rewrite the above Hamiltonian in a picture in which the interaction with the external field has already been diagonalized:

$$
|\bar{\psi}\rangle=e^{i h t}|\psi\rangle, \quad h=S^{+} \mathcal{E}+S^{-} \mathcal{E}^{*},
$$

where $|\bar{\psi}\rangle$ is transformed atomic state in new picture from old atomic state $|\psi\rangle$. The Hamiltonian in this picture is

$$
\begin{aligned}
& \bar{H}=-i g e^{i h t}\left(S^{+} a-S^{-} a^{\dagger}\right) e^{-i h t}, \\
& e^{i h t} \equiv \cos (|\mathcal{E}| t)+\frac{i h}{|\mathcal{E}|} \sin (|\mathcal{E}| t) .
\end{aligned}
$$

The atomic spin operators $S^{ \pm}$transform as 


$$
\begin{aligned}
e^{i h t} S^{+} e^{-i h t} \equiv & S^{+} \cos ^{2}(|\mathcal{E}| t)+\frac{\mathcal{E}^{* 2}}{|\mathcal{E}|^{2}} \sin ^{2}(|\mathcal{E}| t) S^{-} \\
& -\frac{2 i \mathcal{E}^{*}}{|\mathcal{E}|} S^{z} \sin (|\mathcal{E}| t) \cos (|\mathcal{E}| t), \\
e^{i h t} S^{-} e^{-i h t} \equiv & S^{-} \cos ^{2}(|\mathcal{E}| t)+\frac{\mathcal{E}^{2}}{|\mathcal{E}|^{2}} \sin ^{2}(|\mathcal{E}| t) S^{+} \\
& +\frac{2 i \mathcal{E}}{|\mathcal{E}|} S^{z} \sin (|\mathcal{E}| t) \cos (|\mathcal{E}| t) .
\end{aligned}
$$

Using Eqs. (5) and (6), Eq. (3) becomes

$$
\begin{aligned}
\bar{H}= & -i g\left(S^{+} \cos ^{2}(|\mathcal{E}| t)+\frac{\mathcal{E}^{* 2}}{|\mathcal{E}|^{2}} \sin ^{2}(|\mathcal{E}| t) S^{-}\right. \\
& \left.-\frac{2 i \mathcal{E}^{*}}{|\mathcal{E}|^{2}} S^{z} \sin (|\mathcal{E}| t) \cos (|\mathcal{E}| t)\right) a-\text { H.c. }
\end{aligned}
$$

We note that the Hamiltonians of the above form have been previously used to treat the inhibition of the spontaneous emission [9] and for the production of mesoscopic superposition states $[10,11]$. We also note that the eigenstates of the Hamiltonian (1) with $\mathcal{E}$ also treated as a quantized field have been given in [12] and applied to calculate, for example, the spectrum of resonance fluorescence from an atom in a bichromatic field. We assume that the atom is driven strongly so that $|\mathcal{E}|$ is large and hence we drop rapidly oscillating terms from Eq. (7)-i.e., $e^{ \pm 2 i|\mathcal{E}| t} \Rightarrow 0$. Then Eq. (7) reduces to

$$
\bar{H}=-\frac{i g}{2}\left(S^{+}+\frac{\mathcal{E}^{* 2}}{|\mathcal{E}|^{2}} S^{-}\right) a-\text { H.c. }
$$

We choose $\mathcal{E}^{* 2} /|\mathcal{E}|^{2}=1$; in general, this can also be done by adjusting phases with atomic operators. Then Eq. (8) takes the form

$$
\bar{H}_{\mathrm{eff}}=g S^{x}\left(\frac{a-a^{\dagger}}{i}\right) .
$$

Note the appearance of the well-known displacement $D(\alpha)$ $=\left(a^{\dagger} \alpha-a \alpha^{*}\right)$ in Eq. (9). In particular we have the momentum operator (out-of-phase quadrature for the field). Further it should also be noted that $h$ as defined by Eq. (2) commutes

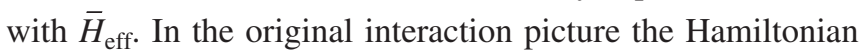
for our model will be

$$
H_{\text {eff }}=g S^{x}\left(\frac{a-a^{\dagger}}{i}\right)+2|\mathcal{E}| S^{x} .
$$

In the effective Hamiltonian (10) the field displacement operator appears with the atomic operator, which can produce a displacement in the field state depending on the atomic state.

\section{REALIZATION OF RANDOM WALKS}

We next discuss the possible realization of quantum random walks in the system of the two-level atom and the field inside the cavity. In Fig. 1 we show a schematic diagram for

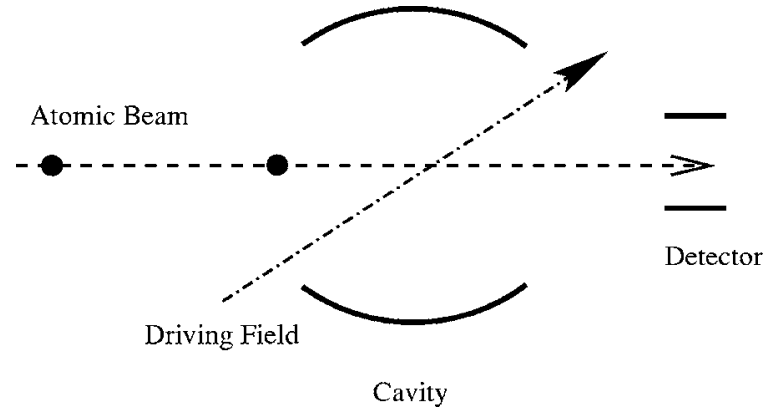

FIG. 1. The schematic arrangements for realizing quantum random walks. A continuous strong driving field inside the cavity can be applied by an external source. The time interval between two atoms in the atomic beam is selected larger than the interaction time in the cavity so that only one atom is presented inside the cavity at a time. The atoms are detected at the exit of the cavity by a stateselective detector.

realizing quantum random walks. In our scheme an atom passes through the cavity and is detected at the exit of the cavity. A continuous strong driving field is applied by using an external source. Let us consider that, initially, the atom is in the superposition state $|\Phi\rangle=\left(c_{1}|e\rangle+c_{2}|g\rangle\right)$ and the field inside the cavity is in a coherent state $|\alpha\rangle$. Using Eq. (10) the combined state of the atom-cavity system after time $t$ is given by

$$
\begin{aligned}
|\psi(t)\rangle= & \exp \left[g t S^{x}\left(a^{\dagger}-a\right)-2 i|\mathcal{E}| t S^{x}\right]|\Phi\rangle|\alpha\rangle \\
= & \frac{c_{+} e^{-i \phi}}{2}(|g\rangle+|e\rangle)|\alpha+g t / 2\rangle \\
& +\frac{c_{-} e^{i \phi}}{2}(|g\rangle-|e\rangle)|\alpha-g t / 2\rangle \\
= & |g\rangle\left[\frac{c_{+} e^{-i \phi}}{2}|\alpha+g t / 2\rangle+\frac{c_{-} e^{i \phi}}{2}|\alpha-g t / 2\rangle\right] \\
& +|e\rangle\left[\frac{c_{+} e^{-i \phi}}{2}|\alpha+g t / 2\rangle-\frac{c_{-} e^{i \phi}}{2}|\alpha-g t / 2\rangle\right], \\
\phi= & \left(|\mathcal{E}|+\frac{g}{2} \operatorname{Im}(\alpha)\right) t,
\end{aligned}
$$

where $c_{+}=c_{1}+c_{2}$ and $c_{-}=c_{1}-c_{2}$. Using normalization of atomic states we can select $c_{-} / c_{+}=\tan \theta$. Thus the detection of the atom in state $|e\rangle$ or $|g\rangle$ leaves the cavity field in a superposition of states $|\alpha+g t / 2\rangle$ and $|\alpha-g t / 2\rangle$. For small values of $g t$ the states $|\alpha+g t / 2\rangle$ and $|\alpha-g t / 2\rangle$ overlap completely, and thus quantum interference effects between $\mid \alpha$ $+g t / 2\rangle$ and $|\alpha-g t / 2\rangle$ become significant. If we assume that the atom is detected in its ground state $|g\rangle$, then the state of the field inside the cavity can be written as

$$
\left|\psi_{f}\right\rangle \propto\left[e^{-i|\mathcal{E}| t} D(g t / 2)+e^{i|\mathcal{E}| t} \tan (\theta) D(-g t / 2)\right]|\alpha\rangle .
$$

Clearly after passing one atom through the cavity the field inside the cavity is displaced backward or forward along the line in a random way by a step of $g t / 2$. We can now iterate 


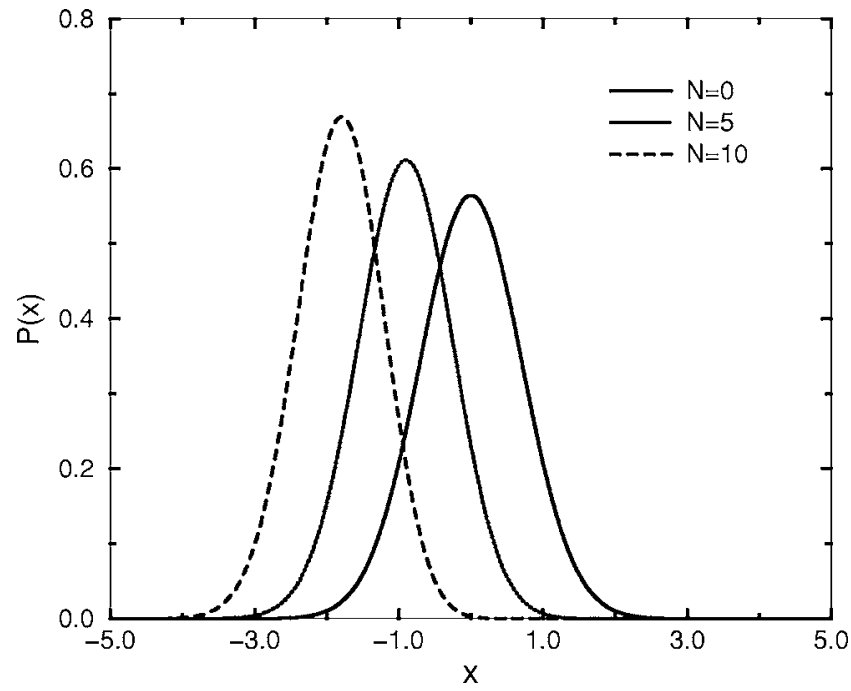

FIG. 2. The probability distribution $P(x)$ for the position of the quantum random walker, assuming the initial wave packet as Gaussian $\exp \left[-(x-\alpha)^{2} / 2\right]$ for $\alpha=0$, step size $l=0.05, \phi=2 \pi$, and $\theta=2 \pi / 3$.

the above step to obtain the state of the field after the passage of $N$ atoms. We assume that atoms enter in the cavity in the state $|\Phi\rangle$ and after interaction with the field inside the cavity detected in their ground state $|g\rangle$. Note that the displacement operators appearing in the above state commute with each other, $[D(g t / 2), D(-g t / 2)]=0$, for real $g t$. Thus the field state after the passage of $N$ atoms is given by

$\left|\psi_{f}(N)\right\rangle=C\left[e^{-i|\mathcal{E}| t} D(g t / 2)+e^{i|\mathcal{E}| t} \tan (\theta) D(-g t / 2)\right]^{N}|\alpha\rangle$

$$
\begin{aligned}
= & C \sum_{m=0}^{N}\left(\begin{array}{l}
N \\
m
\end{array}\right)\left[e^{-i m|\mathcal{E}| t} D^{m}\left(\frac{g t}{2}\right) e^{i(N-m)|\mathcal{E}| t}(\tan \theta)^{N-m}\right. \\
& \left.\times D^{N-m}\left(-\frac{g t}{2}\right)\right]|\alpha\rangle \\
= & C \sum_{m=0}^{N}\left(\begin{array}{l}
N \\
m
\end{array}\right) e^{i(N-2 m)|\mathcal{E}| t}(\tan \theta)^{N-m} D^{N-2 m}(-g t / 2)|\alpha\rangle \\
= & C \sum_{m=0}^{N}\left(\begin{array}{l}
N \\
m
\end{array}\right) e^{i(N-2 m) \phi}(\tan \theta)^{N-m}|\alpha-(N-2 m)(g t / 2)\rangle,
\end{aligned}
$$

where $C$ is normalization constant and we have used the property of the displacement operator $D^{-1}(\alpha)=D(-\alpha)$. On writing the above result in coordinate-space representation, we get the wave function $\psi_{N}(x, \alpha)=\left\langle x \mid \psi_{f}(N)\right\rangle$,

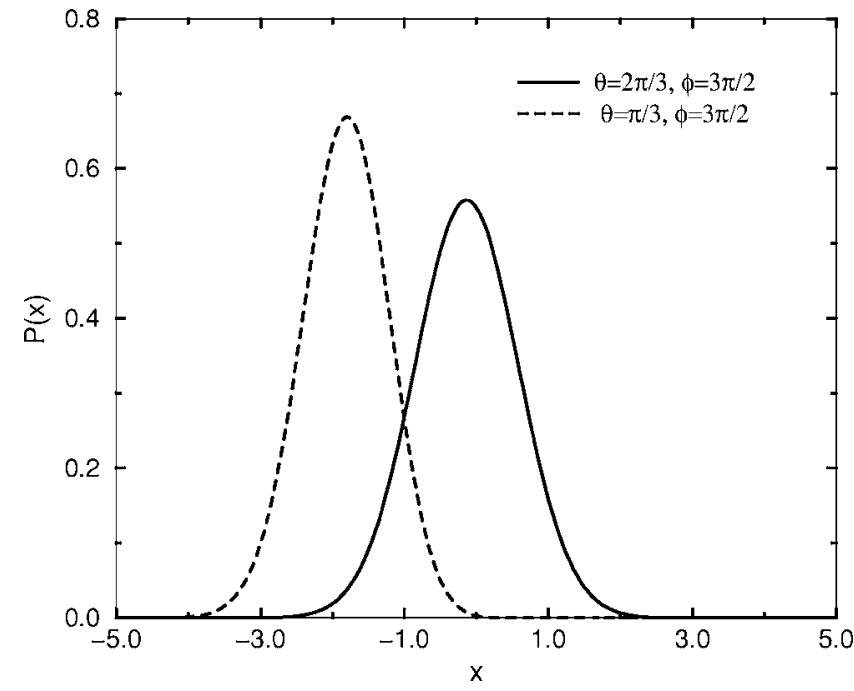

FIG. 3. The probability distribution $P(x)$ for the position of the quantum random walker after ten steps, using different sets of values of $\theta$ and $\phi$ and for $\alpha=0$, step size $l=0.05$.

$$
\psi_{N}(x, \alpha)=C \sum_{m=0}^{N}\left(\begin{array}{l}
N \\
m
\end{array}\right) e^{i(N-2 m) \phi}(\tan \theta)^{N-m} \psi_{\alpha}(x+[N-2 m] l),
$$

where $\psi_{\alpha}(x) \equiv\langle x \mid \alpha\rangle$ is the wave function corresponding to the initial cavity field state $|\alpha\rangle$ which is centered at $x=\alpha$ and the step size of the random walker is $l=g t / 2$. We note that we have recovered the result of Aharonov et al. [1]. In Fig. 2 we have plotted the probability amplitude distribution for the initial wave function $\psi_{\alpha}(x) \sim \exp \left[-(x-\alpha)^{2} / 2\right]$ for real values of $x$ and $\alpha=0$. The displacement depends on $\theta, \phi$, and the number of steps, $N$. The unexpected displacement in the state of the random walker is the result of constructive quantum interference between the states generated in various steps which comes from the off-diagonal terms in $P(x)$ $=\left|\psi_{N}(\alpha, x)\right|^{2}$. We have checked this by dropping the offdiagonal terms in $P(x)$; in that case, $P(x)$ remains same in shape as the initial wave packet but shifts by an amount $\mathrm{Nl}$. The displacement of the random walker is not bounded by the classically possible maximum and minimum displacements $\pm N l$. The quantum interference leads to an arbitrary displacement in the random walker's position and can be much larger than $\pm N l$. A small squeezing in the wave packet is also generated from these interference effects. The selection of phases $\phi$ and $\theta$ is also critical for a displacement in the position of the quantum walker. This can be understood from Eq. (18); each term in Eq. (18) corresponding to a particular value of $m$ represents a possible state of the quantum walker. The final displacement of the walker after $N$ steps comes as a result of quantum interference among all such possible states. Thus the final displacement depends on the relative weights and the relative phases of these states. The relative weights of the states in Eq. (18) are proportional to $(\tan \theta)^{N-m}$ while the relative phases are given by $\phi$. Depending on the values of $\theta$ and $\phi$, the final displacement in the position of the quantum walker can take any value from 

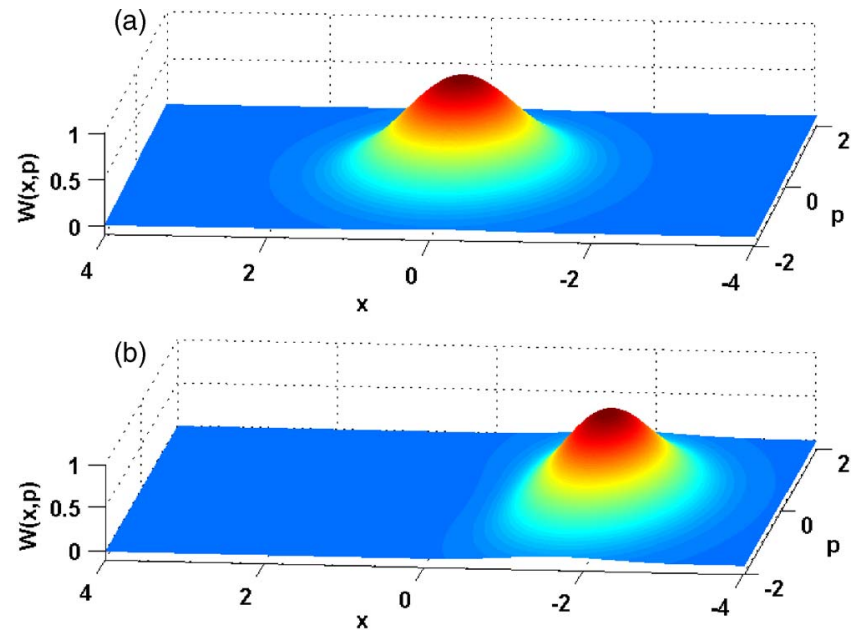

FIG. 4. (Color online) The Wigner function $W(x, p)$ of the state of the random walker, after the number of steps (a) $N=0$ and (b) $N=10$, using the same parameters as in Fig. 2.

the possible maximum to the minimum. For example, for the parameters used in Fig. 2 we plot $P(x)$ using different values of $\theta$ and $\phi$ in Fig. 3. The displacement in the position of the quantum walker is minimum when $\theta=2 \pi / 3$ and $\phi$ is a halfinteger multiple of $\pi$. Further for $\theta=\pi / 3$ and $\phi=3 \pi / 2$ the displacement is maximum again.

For visualizing quantum interferences we plot the Wigner function of the random walker in Fig. 4. The Wigner distribution for any state $\psi(x)$ can be obtained by using the definition [13]

$$
W(x, p)=\frac{1}{\pi \hbar} \int e^{2 i p y / \hbar} \psi(x-y) \psi^{*}(x+y) d y .
$$

In Fig. 4(a) the field is in its initial coherent state and the Wigner function is perfect Gaussian. As the field is displaced by random steps, by passing atoms through the cavity, quantum interference effects start deforming the shape of the Wigner function from the Gaussian. After few steps the Wigner function is squeezed in $x$ quadrature and gets displaced by an arbitrary distance in $x$. In Fig. 4(b) [see also Fig. 6(a)], we have shown the Wigner function after ten random steps for the initial Gaussian wave packet. The squeezing is also clear from Fig. 2 which shows the narrowing of the distribution $P(x)$. It is clear that the displacement in the position of the random walker comes as a result of quantum interference which is a consequence of the quantum coherence between the states generated in random steps.

Here it should be noted that the quantum walks appear as a consequence of quantum interference. Thus maintaining coherence of the system throughout the experiment is an essential requirement. In the context of currently available technologies these requirements can be fulfilled by using Rydberg atoms in a very-high-quality cavity. Such experiments have been successfully carried out by the Paris group $[14,15]$ using open cavities as well as Walther's group [16] using closed cavities. We also note the recent success in trapping atoms in high-quality cavities $[17,18]$. The question is if one can use trapped atoms to realize the quantum random walk instead of flying atoms. We believe that this should be possible by using (i) a trigger pulse, the duration of which would set the interaction time, (ii) the detection of the atomic state possibly by using a very short pulse, and (iii) resetting of the atomic state. In this arrangement the same atom is used repeatedly rather than sending atoms one by one. As a matter of fact some of these ideas are in vogue [19].

\section{MEASUREMENT OF THE STATE OF THE RANDOM WALKER}

We next discuss how we can probe the quantum state of the random walker. We propose homodyne techniques [15] for measuring the state of the random walker. Such homodyne measurements can be performed by mixing an external resonant coherent field to the cavity and then probing the resultant cavity field by passing a test atom through the cavity. In the previous section, we have shown how the cavity field is displaced backward or forward in a random step by passing a single atom through the cavity. The state of the field in the cavity after such $N$ steps can be monitored by homodyne measurements which can be implemented in the same experimental setup. After displacing the field inside the cavity by $N$ random steps, by passing $N$ atoms, a resonant external coherent field $|\beta\rangle$ is injected into the cavity. After adding the external field, the state of the resultant field in the cavity is

$$
\begin{aligned}
\left|\psi_{H}\right\rangle= & C \sum_{m=0}^{N}\left(\begin{array}{l}
N \\
m
\end{array}\right) e^{i(N-2 m) \phi(\tan \theta)^{N-m}} \\
& \times D(\beta)|\alpha-(N-2 m)(g t / 2)\rangle \\
= & C \sum_{n} \sum_{m=0}^{N}\left(\begin{array}{l}
N \\
m
\end{array}\right) e^{i(N-2 m) \phi}(\tan \theta)^{N-m} \\
& \times\langle n|D(\beta)| \alpha-(N-2 m)(g t / 2)\rangle|n\rangle \\
= & \sum_{n} F_{n}|n\rangle, \\
F_{n}= & C \sum_{m=0}^{N}\left(\begin{array}{l}
N \\
m
\end{array}\right) e^{i(N-2 m) \phi(\tan \theta)^{N-m}} \\
& \times\langle n|D(\beta)| \alpha-(N-2 m)(g t / 2)\rangle .
\end{aligned}
$$

Now we bring a similar atom in its lower-energy state $|g\rangle$ to probe the cavity field. The probability of detecting the probe atom in its lower state $|g\rangle$ after crossing the cavity in time $t_{p}$ is

$$
P_{g}=\sum_{n}\left|F_{n}\right|^{2} \cos ^{2}\left(g t_{p} \sqrt{n}\right) .
$$

The interaction time $t_{p}$ for the probe atom is selected such that if there are photons in the cavity, it leaves the cavity in its higher-energy state $|e\rangle$ with larger probability. If we choose the external field $|\beta\rangle$ such that $\beta=-\alpha+\delta$, the probe atom will leave the cavity in its ground state with larger 


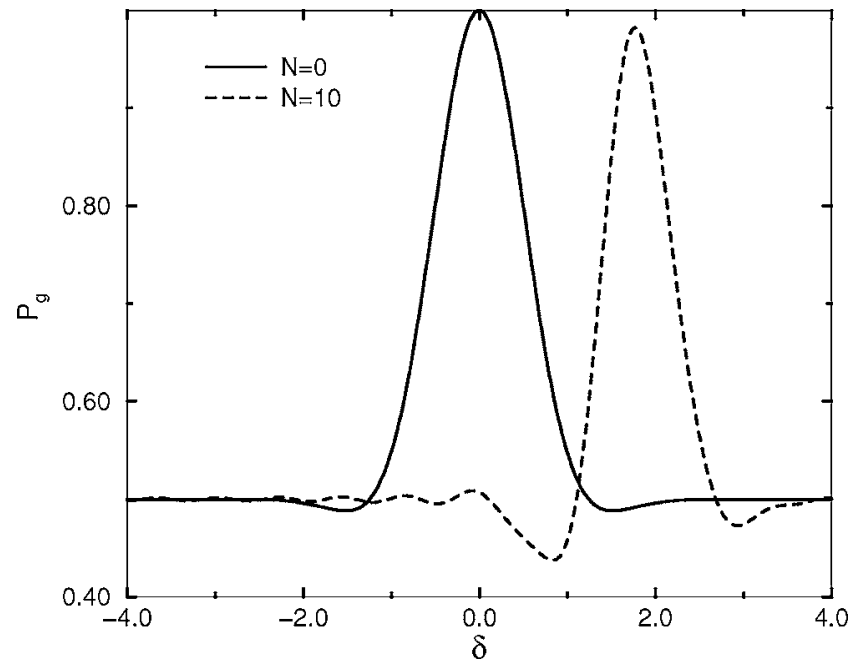

FIG. 5. The probability of detecting the probe atom in its ground state as a function of $\delta$ for the state of the quantum random walker after number of steps, $N=0$ (solid line) and $N=10$ (dashed line). The parameters used are same as in Fig. 2, and the interaction time for the probe atom is selected such that $g t_{p}=1.5 \pi$.

probability when the value of $\delta$ will be opposite and equal to the displacement of the random walker from the initial position $\alpha$. Thus the probability of the probe atom leaving the cavity in its lower state $|g\rangle$ would, as a function of $\delta$, have a peak corresponding to the positions of the random walker after $N$ steps. In Fig. 5, we plot the probability of detecting the probe atom in its lower state with $\delta$. The solid line curve is result of homodyne measurements of the position of the random walker corresponding to its initial state. The dashed line curve corresponds to the homodyne measurement after ten steps using the same parameter as in Fig. 2. Clearly the homodyne measurement yields the state of the quantum walker (Fig. 2). Thus the homodyne measurement can be an elegant way for monitoring the position of the random walker in our model of realizing quantum random walks.

\section{DECOHERENCE OF THE GENERATED STATE OF THE RANDOM WALKER}

Quantum random walks are different from the classical random walks in the sense of quantum interferences which may lead to much larger displacements in the position of the quantum random walker than the classically possible maximum displacements. These quantum interferences are the consequences of coherence in the system. Clearly we need the coherence to live for a long time, and thus it is important to study the effects of the decoherence of the system. In this section we study the decoherence of the state of the random walker due to damping in the cavity. This can be done using the master equation

$$
\dot{\rho}=-\frac{\kappa}{2}\left(a^{\dagger} a \rho-2 a \rho a^{\dagger}+\rho a^{\dagger} a\right),
$$

where $\kappa$ is cavity field decay parameter and we carry the analysis in the absence of thermal photons. For the initial
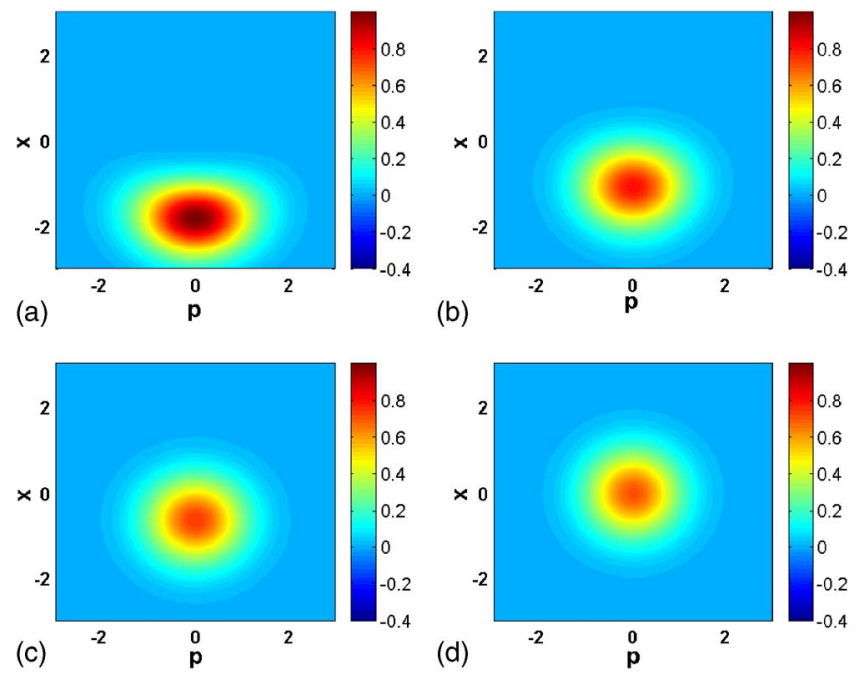

FIG. 6. (Color online) The decoherence of the state of the random walker in terms of the Wigner function at different times, for $N=10, l=0.05, \theta=2 \pi / 3, \phi=2 \pi$, and (a) $\kappa t=0$, (b) $\kappa t=1 / 4 N^{2} l^{2}$, (c) $\kappa t=1 / 2 N^{2} l^{2}$, and (d) $\kappa t=2 / N^{2} l^{2}$.

state (17) we find the density matrix after time $t$ :

$$
\begin{aligned}
\rho(t)= & |C|^{2} \sum_{m=0}^{N} \sum_{n=0}^{N}\left(\begin{array}{l}
N \\
m
\end{array}\right)\left(\begin{array}{l}
N \\
n
\end{array}\right) e^{2 i(n-m) \phi}(\tan \theta)^{2 N-m-n} \\
& \times\langle\alpha-(N-2 m) l \mid \alpha-(N-2 n) l\rangle^{\left(1-e^{-\kappa t}\right)} \\
& \times|\alpha-(N-2 m) l\rangle_{t}\left\langle\alpha-\left.(N-2 n) l\right|_{t}\right.
\end{aligned}
$$

where $|\zeta\rangle_{t} \equiv\left|\zeta e^{-\kappa t / 2}\right\rangle$. In the limit $\kappa t \ll 1$, Eq. (24) simplifies to

$$
\begin{aligned}
\rho(t)= & |C|^{2} \sum_{m=0}^{N} \sum_{n=0}^{N}\left(\begin{array}{l}
N \\
m
\end{array}\right)\left(\begin{array}{l}
N \\
n
\end{array}\right) e^{2 i(n-m) \phi}(\tan \theta)^{2 N-m-n} \\
& \times e^{-2 \kappa t l^{2}(n-m)^{2}}|\alpha-(N-2 m) l\rangle\langle\alpha-(N-2 n) l| .
\end{aligned}
$$

Thus the coherence of the state decays on the time scales $1 / 2 N^{2} l^{2}$. In Fig. 6 we show the decoherence effects due to the cavity damping in the state of the quantum random walker in terms of the Wigner function. As the time progresses from (a) to (d) the decoherence reduces the quantum interference effects and the state of the random walker decays to its initial state. In Fig. 6(a) the Wigner function for the state of the random walker after ten steps using the parameters of Fig. 4(b) is plotted which is squeezed in $x$ quadrature and centered at $x \approx-2$. As a result of decoherence due to cavity damping the quantum interferences start decaying and the Wigner function changes to the perfect Gaussian shape, Fig. 6(c) centered at $x=N l$. Now the field inside the cavity is almost in a coherent state and decays with the cavity damping rate. Further the lifetime for the state of the quantum random walker is given by $T_{N}=T_{c} / 2 N^{2} l^{2}$ where $T_{c}=1 / \kappa$ is the lifetime for field in the cavity. 


\section{CONCLUSIONS}

In conclusion we have shown a simple possible realization of quantum random walks using cavity QED. We have proposed homodyne detection for monitoring the position of the random walker. We have also discussed the decoherence effects and the time scales at which the quantum nature of random walks survives. As a result of new emerging technologies various improved cavities are feasible these days [14-16], which makes our proposal very interesting and realistic. Such a realization of quantum random walks may be useful for implementing various algorithms [20] based on quantum random walks. Finally it should be noted that generalizations of the present work to more than one dimension are possible.

\section{ACKNOWLEDGMENTS}

We acknowledge support from the National Science Foundation Grant No NSFCCF-0524673.

\section{APPENDIX: STATE OF THE WALKER FOR NO MEASUREMENT ON THE ATOMIC STATE}

In this appendix we would like to connect the result (13) explicitly to the case of the classical random walk. For this purpose we find the reduced state of the field from Eq. (13).
We also set $c_{1}=1, c_{2}=0$; then the reduced state of the field $\rho_{f}$ is

$$
\rho_{f}=\frac{1}{2}\left(\left|\alpha+\frac{g t}{2}\right\rangle\left\langle\alpha+\frac{g t}{2}|+| \alpha-\frac{g t}{2}\right\rangle\left\langle\alpha-\frac{g t}{2}\right|\right) .
$$

Clearly the state of the field after the passage of $N$ atoms would be

$$
\begin{aligned}
\rho_{f} & =\left(\frac{1}{2}\right)^{N} \sum_{m=0}^{N}\left(\begin{array}{l}
N \\
m
\end{array}\right)\left|\alpha+\frac{g t}{2}(N-2 m)\right\rangle\left\langle\alpha+\frac{g t}{2}(N-2 m)\right| \\
& =\left(\frac{1}{2}\right)^{N} \sum_{p=-N}^{+N} \frac{N !}{\frac{N-p}{2} ! \frac{N+p}{2} !}\left|\alpha+\frac{g t}{2} p\right\rangle\left\langle\alpha+\frac{g t}{2} p\right|,
\end{aligned}
$$

which is reminiscent of the result for the classical random walk in the sense that the weight factor of the state $\mid \alpha$ $+(g t / 2) p\rangle\langle\alpha+(g t / 2) p|$ is the same as the probability of finding the walker at the site $p$ [21]. It should, however, be borne in mind that the coherent states $|\alpha+(g t / 2) p\rangle$ and $\mid \alpha$ $\left.+(g t / 2) p^{\prime}\right\rangle$ are not orthogonal for $p \neq p^{\prime}$.
[1] Y. Aharonov, L. Davidovich, and N. Zagury, Phys. Rev. A 48, 1687 (1993).

[2] B. C. Sanders, S. D. Bartlett, B. Tregenna, and P. L. Knight, Phys. Rev. A 67, 042305 (2003).

[3] T. Di, M. Hillery, and M. S. Zubairy, Phys. Rev. A 70, 032304 (2004).

[4] B. C. Travaglione and G. J. Milburn, Phys. Rev. A 65, 032310 (2002).

[5] W. Dür, R. Raussendorf, V. M. Kendon, and H.-J. Briegel, Phys. Rev. A 66, 052319 (2002); K. Eckert, J. Mompart, G. Birkl, and M. Lewenstein, e-print quant-ph/0503084.

[6] P. L. Knight, E. Roldan, and J. E. Sipe, Phys. Rev. A 68, 020301(R) (2003); Opt. Commun. 227, 147 (2003).

[7] D. Bouwmeester, I. Marzoli, G. P. Karman, W. Schleich, and J. P. Woerdman, Phys. Rev. A 61, 013410 (2000).

[8] B. Do, M. L. Stohler, S. Balasubramanian, D. S. Elliott, C. Eash, E. Fischbach, M. A. Fischbach, A. Mills, and B. Zwickl, J. Opt. Soc. Am. B, 22, 499 (2005); H. Jeong, M. Paternostro, and M. S. Kim, Phys. Rev. A 69, 012310 (2004).

[9] G. S. Agarwal, W. Lange, and H. Walther, Phys. Rev. A 48, 4555 (1993); W. Lange and H. Walther, ibid. 48, 4551 (1993).

[10] E. Solano, G. S. Agarwal, and H. Walther, Phys. Rev. Lett. 90, 027903 (2003).

[11] P. Lougovski, F. Casagrande, A. Lulli, B.-G. Englert, E. Sol- ano, and H. Walther, Phys. Rev. A 69, 023812 (2004).

[12] H. S. Freedhoff and Z. Ficek, Phys. Rev. A 55, 1234 (1997); Z. Ficek and H. S. Freedhoff, ibid. 53, 4275 (1996).

[13] M. Hillery, R. F. O'Connell, M. O. Scully, and E. P. Wigner, Phys. Rep. 106, 121 (1984).

[14] T. Meunier, S. Gleyzes, P. Maioli, A. Auffeves, G. Nogues, M. Brune, J. M. Raimond, and S. Haroche, Phys. Rev. Lett. 94, 010401 (2005).

[15] A. Auffeves, P. Maioli, T. Meunier, S. Gleyzes, G. Nogues, M. Brune, J. M. Raimond, and S. Haroche, Phys. Rev. Lett. 91, 230405 (2003).

[16] B. T. H. Varcoe, S. Brattke, M. Weidinger, and H. Walther, Nature (London) 403, 743 (2000); M. Keller, B. Lange, K. Hayasaka, W. Lange, and H. Walther, ibid. 431, 1075 (2004).

[17] A. Boca, R. Miller, K. M. Birnbaum, A. D. Boozer, J. Mc Keever, and H. J. Kimble, Phys. Rev. Lett. 93, 233603 (2004).

[18] P. Maunz, T. Puppe, I. Schuster, N. Syassen, P. W. H. Pinkse, and G. Rempe, Nature (London) 428, 50 (2004).

[19] T. Legero, T. Wilk, M. Hennrich, G. Rempe, and A. Kuhn, Phys. Rev. Lett. 93, 070503 (2004).

[20] N. Shenvi, J. Kempe, and K. Birgitta Whaley, Phys. Rev. A 67, 052307 (2003).

[21] S. Chandrasekhar, Rev. Mod. Phys. 15, 1 (1943). 